\title{
TODCOR: A Two-Dimensional Correlation of a Composite Spectrum to Derive the Radial Velocities of its Components
}

\author{
TSEVI MAZEH \& SHAY ZUCKER \\ School of Physics and Astronomy, Tel-Aviv University, \\ Tel Aviv 69978, Israel
}

Cross correlation is a frequently used technique to obtain the Doppler shifts of digitized celestial spectra. This method, suggested by Tonry \& Davis (1979), cross correlates the observed spectrum against an assumed template, and obtains the stellar radial velocity by the location of the correlation maximum (Wyatt 1985). The technique finds the correct radial velocity even for extremely low $\mathrm{S} / \mathrm{N}$ spectra.

Spectra composed of two components present a potential difficulty to this technique. The cross correlation of these spectra usually displays a double peak which can not be resolved whenever the relative velocity of the two components is small. To overcome this difficulty, we developed TODCOR - a new TwO-Dimensional CORrelation algorithm which can simultaneously derive the Doppler shifts of the two components.

TODCOR assumes that the observed spectrum is a combination of two known spectra with unknown shifts. Following the one-dimensional technique, the algorithm calculates the correlation of the observed spectrum against a set of combinations of two templates, with all possible shifts. The correlation, thus, is a two-dimensional function, whose two independent variables are the radial velocities of the two components. The location of the maximum of this function corresponds to the actual Doppler shifts of the two components.

A straight forward implementation of the algorithm would require an enormous amount of computation, turning the approach impractical with the present computers. Therefore, we developed an efficient method to perform the algorithm, reducing the computation needed to analyze spectra containing a thousand points by three orders of magnitude.

The two radial velocities are independent variables, and therefore there is no special significance to their difference. Consequently, TODCOR is able, in princliple, to resolve even components of equal velocities. To test the algorithm, we applied it to a series of simulated spectra composed of calculated stellar spectra of different spectral types, including cases where the velocities of the two components were identical. We found, indeed, that TODCOR yielded accurately the correct results even in those cases.

To demonstrate the capability of TODCOR we present here an analysis of one spectrum composed of calculated A-star and G-star type spectra (Kurucz 1992), with intensity ratio of 0.25 . The rotational velocities of the two spectra are $20 \mathrm{~km} \mathrm{~s}^{-1}$ each, and their Doppler shifts were chosen as 0 and $39 \mathrm{~km} \mathrm{~s}^{-1}$, respectively. This is a velocity difference which is about twice the line width of each of the two components, and therefore barely resolvable. To simulate a real observed spectrum, a Gaussian noise with $\mathrm{S} / \mathrm{N}$ of 20 was added.

For comparison, we applied first the one-dimensional cross correlation tech- 

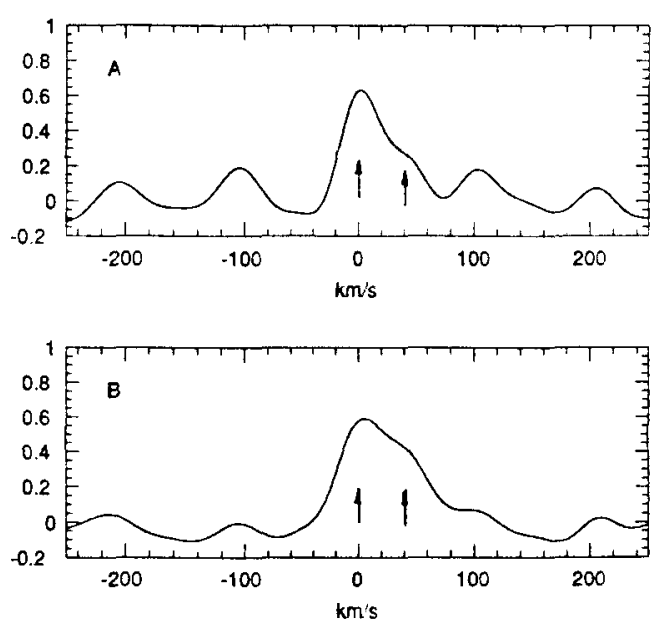

FIGURE 1. The one-dimensional cross correlation functions of the composite spectrum against the two templates. The arrows indicate the velocities used in the simulation.

nique to the composite spectrum, using as template each of the two original spectra. The results are presented in Figure 1.

Evidently, the existence of two individual peaks in the cross correlation can be seen in both correlations, but they are very difficult to resolve quantitatively.

The obtained two-dimensional coorelation function has a very pronounced maximum located at velocities of 0.2 and $40.0 \mathrm{~km} \mathrm{~s}^{-1}$. Figure 2 presents the contour plot of the function around this maximum.

Figure 3 displays cross sections of the same function along the dashed lines drawn in Figure 2.

The curves of Figure 3 are actually one-dimensional cross correlations of

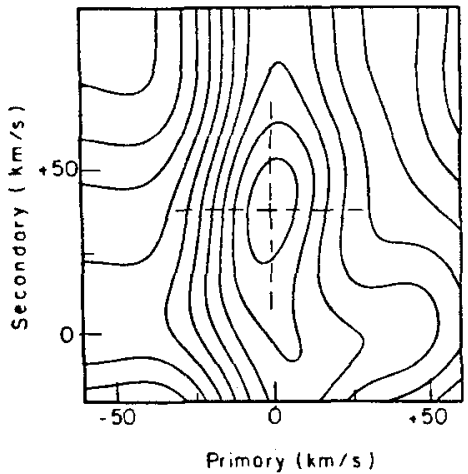

FIGURE 2. A contour plot of the two-dimensional correlation function around the maximum. The dashed lines indicate the lines along which the curves in Figure 3 were taken. 

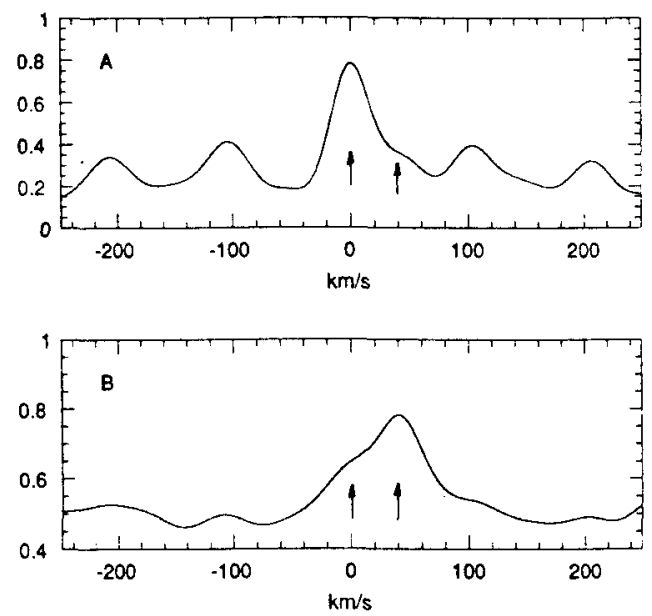

FIGURE 3. Cross sections of the two-dimensional correlation function, taken along the dashed lines of Figure 2. The arrows indicate the velocities used in the simulation.

the composite spectrum against each of the templates, taking into account the effect of the other template. The pronounced peaks indicate the high significance detection of the two velocities.

One of the immediate applications of TODCOR is, obviously, the measurement of the radial velocity curves of spectroscopic binaries. In particular, TODCOR is advantageous when the two components are of comparable intensity and the the relative velocity is small. Moreover, TODCOR can obtain the radial velocity of a faint secondary spectrum, even if its one-dimensional cross correlation peak cannot be detected. This feature can turn a substantial part of the known single-lined binaries into double-lined ones.

\section{REFERENCES}

Kurucs, R.L. 1992, in Precision Photometry: Astrophysics of the Galasy, ed. A.G.D. Philip, A.R. Upgren, and K.A. Janes (Schenectady, New York), in press

Tonry, J. \& Davis, M. 1979, AJ, 84, 1511

Wyatt, W.F. 1985, in IAU Colloquium 88, Stellar Radial Velocities, ed. A.G.D. Philip and D.W. Latham (Schenectady, New York), p. 123 